\title{
Super-resolution Ultrasound Imaging of the Renal Microvasculature in Rats with Metabolic syndrome
}

Søgaard, Stinne Byrholdt; Andersen, Sofie Bech; Taghavi, Iman; Villagómez Hoyos, Carlos Armando; Hansen, Kristoffer Lindskov; Gran, Fredrik; Jensen, Jørgen Arendt; Nielsen, Michael Bachmann; Sørensen, Charlotte Mehlin

Published in:

2020 IEEE International Ultrasonics Symposium

Link to article, DOI:

10.1109/IUS46767.2020.9251652

Publication date:

2020

Document Version

Peer reviewed version

Link back to DTU Orbit

Citation (APA):

Søgaard, S. B., Andersen, S. B., Taghavi, I., Villagómez Hoyos, C. A., Hansen, K. L., Gran, F., Jensen, J. A., Nielsen, M. B., \& Sørensen, C. M. (2020). Super-resolution Ultrasound Imaging of the Renal Microvasculature in Rats with Metabolic syndrome. In 2020 IEEE International Ultrasonics Symposium IEEE. https://doi.org/10.1109/IUS46767.2020.9251652

\section{General rights}

Copyright and moral rights for the publications made accessible in the public portal are retained by the authors and/or other copyright owners and it is a condition of accessing publications that users recognise and abide by the legal requirements associated with these rights.

- Users may download and print one copy of any publication from the public portal for the purpose of private study or research.

- You may not further distribute the material or use it for any profit-making activity or commercial gain

- You may freely distribute the URL identifying the publication in the public portal 


\title{
Super-resolution Ultrasound Imaging of the Renal Microvasculature in Rats with Metabolic syndrome
}

\author{
Stinne Byrholdt Søgaard ${ }^{1,2,3}$, Sofie Bech Andersen ${ }^{1,2,3}$, Iman Taghavi ${ }^{4}$, Carlos Armando Villagómez Hoyos ${ }^{5}$, Kristoffer Lindskov \\ Hansen ${ }^{1,3}$, Fredrik Gran ${ }^{5}$, Jørgen Arendt Jensen ${ }^{4}$, Michael Bachmann Nielsen ${ }^{1,3}$, Charlotte Mehlin Sørensen ${ }^{3}$. \\ ${ }^{1}$ Department of Diagnostic Radiology, Rigshospitalet, Copenhagen, Denmark. \\ ${ }^{2}$ Department of Biomedical Sciences, University of Copenhagen, Copenhagen, Denmark. \\ ${ }^{3}$ Department of Clinical Medicine, University of Copenhagen, Copenhagen, Denmark. \\ ${ }^{4}$ Center for Fast Ultrasound Imaging, Department of Health Technology, Technical University of Denmark, Lyngby, Denmark. \\ ${ }^{5}$ BK Medical, Herlev, Denmark.
}

\begin{abstract}
Super-resolution ultrasound imaging (SRI) can visualize and quantify changes in the microvasculature. Metabolic syndrome is associated with hyperlipidemia that affect different organs, including the kidneys. Ex vivo studies have shown glomerular injury in Obese Zucker rats (OZR) over time. If in vivo SRI can diagnose renal disease before it becomes evident with current measures, earlier treatment can be initiated to postpone the onset of renal complications in persons with metabolic syndrome. The overall aim of this study is to investigate whether SRI can detect early microvascular changes in the kidneys of rats with metabolic syndrome. The rats presented in this work were scanned at an early age to get a baseline scan prior to further studies. In this work an 11-week-old OZR and a healthy agematched Zucker rat were investigated. During open surgery, the left kidney was scanned for 10 min using a modified BK5000 scanner (BK Medical, Denmark), a fixed X18L5s transducer and intravenously administered SonoVue (1:10). Images were obtained using interleaved contrast (amplitude modulation) and B-mode sequences with focused beam transmission $(6 \mathrm{MHz}, 50 \mathrm{~Hz}$, MI: 0.2). An in-house tool was used to track microbubble (MB) movements between frames, in order to estimate the MB velocities, which were measured in a large region of the cortex and the outer medulla. This is the first time SRI has been used on the kidneys of rats with Metabolic syndrome. Both the cortex and the medulla were well-perfused with MBs, and no morphological differences in the microvasculature were found between the two rats. The thickness of the cortex and the medulla was almost identical; cortex $1.8 \mathrm{~mm}$, medulla $8 \mathrm{~mm}$, craniocaudal length $2.0 \mathrm{vs} .1 .9 \mathrm{~cm}$ (healthy vs. OZR). The same was true regarding the MB velocities (median (IQR) in $\mathrm{mm} / \mathrm{s}$ ) for healthy vs. OZR; cortex 0.75 (3.51) vs. $0.65(2.64)$ and medulla $0.75(0.32)$ vs. $0.62(0.30)$. The results show that SRI can visualize the renal microvasculature of a young OZR. Therefore, the method can be used for further investigations of the renal microvascular changes that occur in the course of Metabolic syndrome.
\end{abstract}

Keywords-Super-resolution ultrasound imaging, OZR, renal disease

\section{INTRODUCTION}

Super-resolution ultrasound imaging (SRI) is a new US technique that makes it possible to visualize the microvasculature in vivo. Studies have shown the ability to do in vivo SRI of organs and tissues in different animals, but the literature on pathological changes is still sparse [1]-[6]. Obese Zucker Rats (OZR) has a mutation in the leptin receptor gene which results in obesity, hyperinsulinemia, poorly regulated blood glucose giving them hyperlipidemia and glomerulosclerosis at an early age. The rat-model develop albuminuria as the one measurable factor, as seen in correlation with diabetes. [7], [8] Diabetes Mellitus (DM) is associated with microvascular disease in several organs including the kidneys. Microalbuminuria (excretion of 30-300 mg/24 hours of albumin in the urine) is the earliest measurable stage of microvascular kidney disease in DM. Progressing into macroalbuminuria it can manifest as diabetic nephropathy (DN), defined by urinary albumin excretion $>300 \mathrm{mg} / 24$ hours. $\mathrm{DN}$ is one of the main causes of end-stage renal failure and results in a poor prognosis for the patients and high medical cost [9]. Since the kidneys have a rich vascularity and because microvascular dysfunction greatly affects kidney function, SRI of the kidneys has a big potential. With our current measures we cannot predict who develops diabetic nephropathy until DN is manifest. Therefore, our vision is to detect early changes in the renal microvasculature. SRI uses gas-filled microbubbles (MBs) as intravascular tracers, allowing imaging of the microvascular anatomy as well as estimation of MB flow velocity and direction [1], [2].

\section{MATERIALS AND METHODS}

\section{A. Animals}

The experiment was conducted on one 11-week-old OZR and one healthy age-matched Zucker rat (396 and 308g). The experiments were executed according to protocols approved by the Danish National Animal Experiments Inspectorate. The procedures were performed at the University of Copenhagen, and all local ethical standards were respected. The ethical policy of the university is consistent with that of the National Institutes of Health (NIH). The rats were obtained at 9 weeks, fed standard laboratory chow ad libitum, allowed free access to water and housed in an animal facility at the University of Copenhagen, Department of Experimental Medicine, under the responsibility of trained animal caretakers. Prior to the scans the rats were anesthetized with $5 \%$ isoflurane and placed on a heating table to maintain normal body temperature $\left(37^{\circ} \mathrm{C}\right)$. The blood pressure was measured continuously by a catheter in the carotid artery. After initial anesthesia a final isoflurane concentration of $1-2 \%$ through the tracheostomy was maintained. The rat's ventilation was controlled by a mechanical ventilator with a respiration cycle of 72 respirations/min. After laparotomy, to further expose the left kidney and reduce the respiratory motion, a metal retractor under the left diaphragm pulling slightly cranially was 


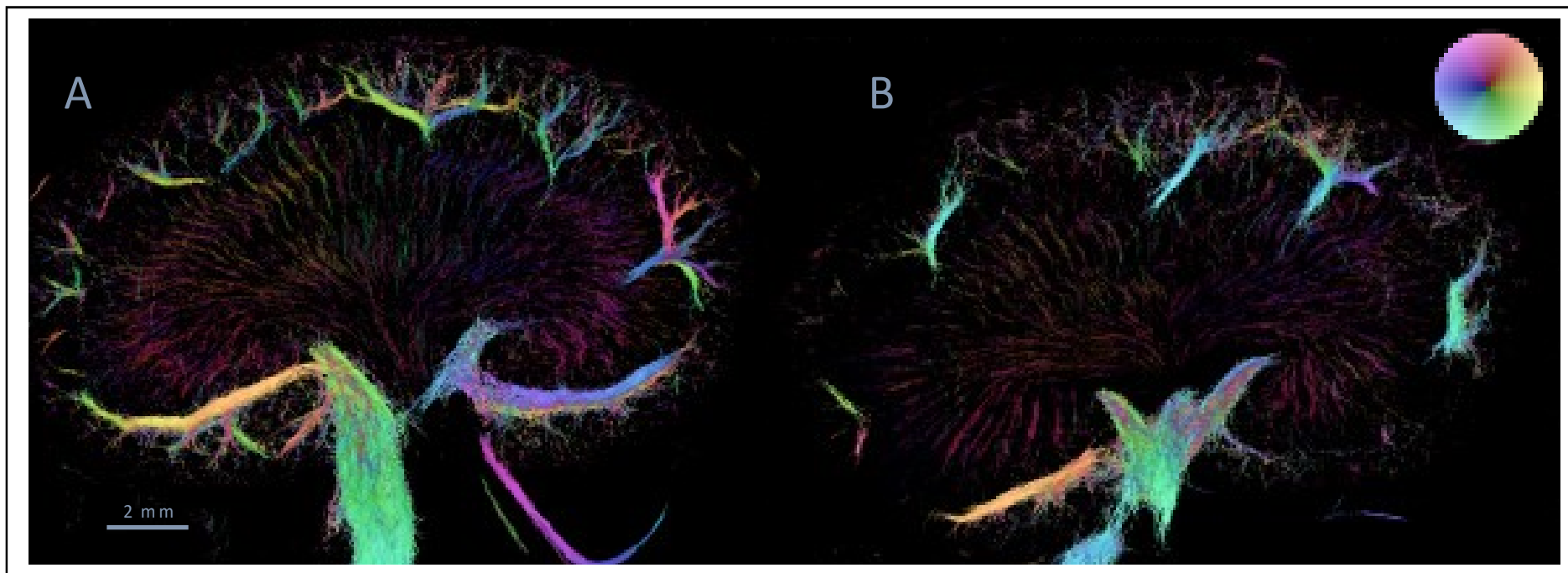

Fig.1 These images is filtered by including every track, Hierarchical-Kalman tracker and motion compensation applied, MB lifetime 3, maximum velocity 15 (mm/sec). A: Microbubble velocity image of an 11-week-old healthy Zucker rat, showing the microbubble flow directions according to the color wheel in the top right corner.

B: Microbubble velocity image of an 11-week-old OZR.

established. Prior to every scan the rat was infused with Nimbex (cisatracurium, $0.85 \mathrm{mg} / \mathrm{ml}$, GlaxoSmithKline). Before the scan, blood and urine samples were collected. After the scans the rats were euthanized in anesthesia.

\section{B. Ultrasound scan procedure and SRI technique}

After laparotomy we scanned the left kidney using a modified BK5000 scanner and a fixed X18L5s transducer (BK Medical, Aps, Herlev, Denmark). Sonovue (Bracco, Italy) was used as contrast. The MBs were diluted in isotonic saline (1:10) and injected through a jugular vein catheter. Each SRI scan lasted 10 minutes in order to acquire enough data to visualize the entire vascular bed. [10] The non-linear behavior of the MBs excited by contrast-enhanced sequences (amplitude modulation) with line-per-lined focused beam transmission (frame rat: $50 \mathrm{~Hz}$, center frequency: $6 \mathrm{MHz}$, mechanical index: 0.2) made it possible to obtain the images. Before the tracking of the MBs motion correction was applied [11]. The MB tracks were made by connection of neighboring MBs in sequential images and with the use of Hierarchical-Kalman tracker [12] To estimate the vessels in the ROI the images was filtered by direction, MB lifetime and track length.

\section{Blood and urinary parameters}

Blood samples were collected to measure blood glucose, creatinine and urea. Blood glucose was measured prior to and after the laparotomy, and before euthanasia. Urine was collected over a period of $15 \mathrm{~min}$ for analysis of albumin and creatinine. Both samples were collected to determine the kidney function.

\section{Histology}

After euthanasia the left kidney was removed to document any possible tissue damage by staining. The kidneys were dropped in a glass with $4 \%$ paraformaldehyde and stained by standard hematoxylin and eosin (H\&E), Sirius Red and Silver Jones staining. The images were analyzed blinded by a trained anatomist.

\section{RESULTS}

\section{A. The microvasculature of the rats}

The thickness of the cortex and the medulla was almost identical. The cortex measuring $1.8 \mathrm{~mm}$, the medulla $8 \mathrm{~mm}$ and a craniocaudal length of 2.0 versus $1.9 \mathrm{~cm}$ (healthy versus OZR). The same was the case regarding the MB velocities (median (IQR) in $\mathrm{mm} / \mathrm{s}$ ) for healthy versus OZR. cortex 0.75 (3.51) vs. 0.65 (2.64) and medulla $0.75(0.32)$ vs. $0.62(0.30)$. (fig. 1$)$

The histology (fig 2) showed normal glomeruli both regarding the OZR as well as the healthy rat.

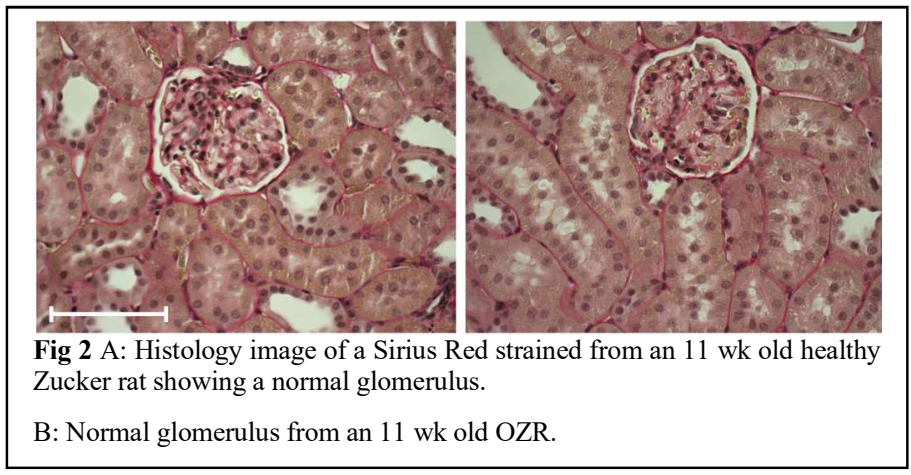

\section{B. Kidney function}

Average blood pressure for the healthy Zucker rat was 62,82 $\mathrm{mmHg}$ versus $94,7 \mathrm{mmHg}$ for the OZR. Blood glucose prior to the scan was $8.3 \mathrm{mmol} / \mathrm{L}$ for the healthy and $9 \mathrm{mmol} / \mathrm{L}$ for the OZR ( $<11 \mathrm{mmol} / \mathrm{L}$ is normal blood glucose).

\section{DISCUSSION}

The histology supported our SRI results showing no morphological differences in the microvasculature between the two rats. The OZR might be too young to see a vascular difference before very late in the illness [13] With this study we would like to move further with SRI by scanning the kidneys of 
rats with metabolic syndrome at a later stage and characterize the microvascular damage caused by metabolic diasease. Another possibility is to use another rat-model; Zucker Diabetic Fatty develop glomerulosclerosis at a much earlier stage. [14], [15]

\section{Acknowledgment (Heading 5)}

The preferred spelling of the word "acknowledgment" in America is without an "e" after the "g". Avoid the stilted expression "one of us (R. B. G.) thanks ...". Instead, try "R. B. G. thanks...". Put sponsor acknowledgments in the unnumbered footnote on the first page.

\section{REFERENCES}

[1] C. Errico et al., "Ultrafast ultrasound localization microscopy for deep super-resolution vascular imaging.," Nature, vol. 527, no. 7579, pp. 499-502, Nov. 2015.

[2] K. Christensen-Jeffries, R. J. Browning, M.-X. Tang, C. Dunsby, and R. J. Eckersley, "In Vivo Acoustic Super-Resolution and SuperResolved Velocity Mapping Using Microbubbles," IEEE Trans. Med. Imaging, vol. 34, no. 2, pp. 433-440, Feb. 2015.

[3] S. B. Andersen et al., "Super-Resolution Ultrasound Imaging of Rat Kidneys before and after Ischemia-Reperfusion.” IEEE, 2019

[4] J. Foiret, H. Zhang, T. Ilovitsh, L. Mahakian, S. Tam, and K. W. Ferrara, "Ultrasound localization microscopy to image and assess microvasculature in a rat kidney," Sci. Rep., vol. 7, no. 1, p. 13662, Dec. 2017.

[5] J. Yu, L. Lavery, and K. Kim, "Super-resolution ultrasound imaging method for microvasculature in vivo with a high temporal accuracy," Sci. Rep., vol. 8, no. 1, p. 13918, Dec. 2018.

[6] Q. Chen, J. Yu, B. M. Rush, S. D. Stocker, R. J. Tan, and K. Kim, "Ultrasound super-resolution imaging provides a noninvasive assessment of renal microvasculature changes during mouse acute kidney injury," Kidney Int., Mar. 2020.

[7] P. G. Schmitz, M. P. O’Donnell, B. L. Kasiske, S. A. Katz, and W. F. Keane, "Renal injury in obese Zucker rats: Glomerular hemodynamic alterations and effects of enalapril," Am. J. Physiol. Ren. Fluid Electrolyte Physiol., vol. 263, no. 3 32-3, 1992.

[8] B. L. Kasiske, M. P. O'Donnell, M. P. Cleary, and W. F. Keane, "Treatment of hyperlipidemia reduces glomerular injury in obese Zucker rats," Kidney Int., vol. 33, no. 3, pp. 667-672, Mar. 1988.

[9] M. Narres et al., "The Incidence of End-Stage Renal Disease in the Diabetic (Compared to the Non-Diabetic) Population: A Systematic Review," PLoS One, vol. 11, no. 1, p. e0147329, Jan. 2016.

[10] V. Hingot, C. Errico, B. Heiles, L. Rahal, M. Tanter, and O. Couture, "Microvascular flow dictates the compromise between spatial resolution and acquisition time in Ultrasound Localization Microscopy," Sci. Rep., vol. 9, no. 1, p. 2456, Dec. 2019.

[11] J. A. Jensen, S. B. Andersen, C. A. V. Hoyos, K. L. Hansen, C. M. Sørensen, and M. B. Nielsen, "Tissue Motion Estimation and Correction in Super Resolution Imaging," in Proc. IEEE Ultrason. Symp., 2019, pp. 1-4.

[12] I. Taghavi et al., "In vivo Ultrasound Super Resolution Imaging with Motion Correction and Robust Tracking", 2020, unpublished.

[13] R. Iliescu and A. R. Chade, "Progressive renal vascular proliferation and injury in obese zucker rats," Microcirculation, vol. 17, no. 4, pp. 250-258, May 2010.

[14] G. J. Etgen and B. A. Oldham, "Profiling of Zucker diabetic fatty rats in their progression to the overt diabetic state.," Metabolism., vol. 49, no. 5, pp. 684-8, May 2000.

[15] N. Yokoi et al., "A novel rat model of type 2 diabetes: The zucker fatty diabetes mellitus ZFDM rat," J. Diabetes Res., vol. 2013, 2013. 